\title{
Prediction of two haptic illusions from the differential adaptation theory
}

\author{
JOAN R. MOORE, KAREN N. JONES, and CHARLES F. GETTYS \\ University of Oklahoma, Norman, Oklahoma 73019
}

\begin{abstract}
A differential adaptation theory was proposed previously to explain two haptic illusions (Jones, Touchstone, \& Gettys, 1974). In the present paper, this theory was used to produce two new illusions. All four illusions can be explained by the notion of differential adaptation caused by intermittent stimulation to one area of the skin and constant stimulation to adjacent areas.
\end{abstract}

A theory of differential adaptation was proposed by Jones, Touchstone, and Gettys (1974) to account for two haptic illusions, the "hourglass" illusion and an illusion reported by Cormack (1973). A weak version of the hourglass illusion can be readily experienced. A rodshaped object, such as a piece of chalk, is held in the center between the thumb and forefinger. If the chalk is smoothly turned end for end, using the fingers of the other hand, a reduction that increases over time will be noticed in the diameter of the chalk at the center of rotation. If the rotation of the chalk is stopped, or if the chalk slips in the fingers, the illusion of an hourglass disappears. Under more controlled conditions, when the rod is turned smoothly by an apparatus, the reduction in the apparent diameter is far greater, and the rod's apparent diameter may actually decrease to the point at which it seems to be about to "pinch in two."

Jones et al. (1974) attributed the hourglass illusion and Cormack's (1973) illusion to "differential adaptation" of adjacent areas of the skin resulting from different rates of adaptation to constant and intermittent stimulation, and they argued that the time course of these illusions is similar to the time course of adaptation of the skin to constant pressure (Kenshalo, 1971). In the hourglass illusion, the skin in contact with the rod at the center of rotation receives relatively constant pressure and adapts quickly, resulting in a decreased sensation of pressure at the center. Skin that is distant from the center of rotation receives intermittent stimulation and so adapts more slowly, or not at all, resulting in the continued perception of pressure. This pressure pattern is translated into the illusion of an "hourglassshaped" object, since the pressure pattern of the rotating stimulus is the same as a fixed hourglass-shaped object.

The experiment reported as "The Ridge Illusion" was conducted by the senior author as a project in an undergraduate advanced experimental psychology class taught by W. Alan Nicewander. The bump illusion data were collected by the junior authors. Reprint requests should be sent to Charles Gettys, Department of Psychology, University of Oklahoma, Norman, Oklahoma 73019.
The purpose of the present paper is to briefly report two additional illusions that were produced by using the principles of the differential adaptation theory. This theory assumes that differences in the intermittency of pressure stimulation to the skin produce differences in pressure adaptation that are perceived as differences in shape. Therefore, an illusion should be produced when one area of the skin is deformed by a constant stimulus and an adjacent area is deformed by an intermittent stimulus. The resulting shape perception should be that of an object, where the relative thickness perceived at the constantly stimulated area is less than at the adjacent intermittently stimulated area. As Jones et al. (1974) previously had made an exhaustive study of the magnitude and the time course of the hourglass illusion, the purpose in the present experiment was solely to demonstrate that new illusions could be discovered from an application of differential adaptation theory.

\section{THE RIDGE ILLUSION}

\section{Method}

Apparatus. The first stimulus presented a constantintermittent-constant pattern of stimulation to adjacent areas of the skin. A flat Plexiglas disk with a circle of cone-shaped "dimples" on each side of the disk was constructed. If the thumb and index finger are placed in contact with both sets of dimples on the disk while the disk is rotated like a wheel, the differential adaptation theory predicts that the dimples will be perceived as raised ridges rather than depressions or indentations.

The disk was $99.6 \mathrm{~mm}$ in diameter and $6.4 \mathrm{~mm}$ thick. Twelve cone-shaped indentations were evenly spaced in a circle $72.3 \mathrm{~mm}$ in diameter on each surface of the disk. Each dimple was $6.6 \mathrm{~mm}$ in diameter and $2.26 \mathrm{~mm}$ deep. A schematic drawing of this stimulus is shown in Figure 1A. The disk was coated with light oil to reduce friction, and all of the apparatus was concealed from the subject's view.

Procedure. The 20 naive subjects grasped the moving disk, rotating at $600 \mathrm{rpm}$, between the thumb and index finger of the preferred hand, placing the thumb over the dimples on one side of the disk and the index finger similarly on the other side. The experimenter guided the subjects' fingers into place, requested that the subjects apply light pressure, and asked if the rough areas (the dimples) were ridges or grooves compared with the smooth areas of the disk. After the verbal report, the subjects were given a card, on which were drawn two parallel lines 


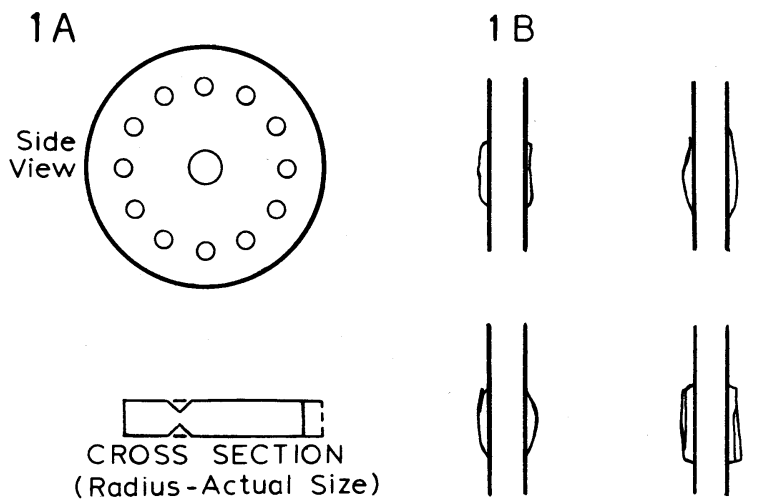

Figure 1. (A) A schematic drawing of the stimulus used in the ridge illusion and (B) drawings of this illusion produced by four subjects.

$6.4 \mathrm{~mm}$ apart, and they were asked to draw the shape of the rough areas using the drawing technique of Von Békésy (1967).

\section{Results}

Eighteen of the 20 subjects reported that they perceived the rough (dimpled) areas as ridges rather than grooves $(\mathrm{p}<.001$, binomial distribution). Measurements of the subjects' drawings of the illusory ridges had a range of $.64-10.39 \mathrm{~mm}$ for the total height for both ridges (mean $=9.96 \mathrm{~mm}, \mathrm{SD}=2.37 \mathrm{~mm}$ ). Four randomly selected drawings are shown in Figure 1B. These drawings are representative of the 18 subjects who experienced the illusion.

\section{Discussion}

The ridge illusion more than doubled the apparent thickness of the disk and was the first illusion discovered from an a priori application of the differential adaptation theory. This success prompted us to try again.

\section{THE BUMP ILLUSION}

The second stimulus was radically different from the stimulus used for the ridge illusion in both shape and speed of rotation. The stimulus used for the bump illusion was a laminate or sandwich consisting of three thicknesses of Plexiglas. Two "half-moons" were sandwiched between two disks (see Figure 2A). If a subject's thumb is pressed against the edge of this revolving laminate, it receives a constant-intermittent-constant pattern of pressure with constant stimulation from the outer disks and intermittent stimulation from the halfmoons. As with the ridge illusion, the differential adaptation theory predicts that the surface that exerts intermittent pressure will be perceived as being raised from the other surface, or in this case, as a "bump."

\section{Method}

Apparatus. The outer disks of the laminate were $59 \mathrm{~mm}$ in diameter, $4.5 \mathrm{~mm}$ thick, and mounted on a shaft $4.5 \mathrm{~mm}$ apart. The inner half-moons were cut from a disk $46.6 \mathrm{~mm}$ in diameter and were mounted between the full outer disks. After the laminate was glued, it was "trued" in a lathe. This operation had the effect of giving each of the half-moons an effective diameter of $59 \mathrm{~mm}$ for $2.29 \mathrm{~cm}$ of the circumference of the laminate, or $45 \mathrm{deg}$ of arc. This arrangement resulted in the edge of the half-moons being flush with the edges of the outer disks part of the time and lower than the edges (or absent) the rest of the time. The laminate was mounted in the apparatus described by Jones et al. (1974).

Procedure. The 14 naive subjects were told to touch the concealed laminate with the ball of their thumbs. It was explained that they would feel an object between the two disks (i.e., the half-moons) and that they were to report whether the object felt higher or lower than the edge of the outer disks. The experimenter guided the subject's thumb into contact with the edge of the motionless laminate for the subject's safety. When the thumb was in place, the laminate was revolved at $30 \mathrm{rpm}$. Each subject had two trials, a 5-sec trial and a $30-\mathrm{sec}$ trial, presented in counterbalanced order. After each trial the subject drew a sketch of his or her shape perceptions on cards on which there were drawings of the side view of the disks.

\section{Results}

All 14 subjects reported an illusory bump at the point that the half-moons contacted their thumbs. The mean height of the illusory bump was calculated to be $3.48 \mathrm{~mm}$ for the 5 -sec trials and $4.05 \mathrm{~mm}$ for the $30-\mathrm{sec}$ trials. This difference was not significant. Four randomly selected drawings made by the subjects are shown in Figure 2B.

\section{GENERAL DISCUSSION}

The differential adaptation theory, which was developed as a post hoc explanation for two haptic illusions in Jones et al. (1974), was used in this paper to discover two additional illusions that were predicted to exist from the theory on a priori grounds. All four illusions studied are compelling and are experienced by nearly all subjects. The mean magnitudes of the two illusions reported by Jones et al. were $2.1 \mathrm{~mm}$ and $1.7 \mathrm{~mm}$. In the present study, the ridge illusion had a mean magnitude of $9.96 \mathrm{~mm}$, and the bump illusion had mean magnitudes of $3.48 \mathrm{~mm}$ and $4.05 \mathrm{~mm}$ for the 5- and 30 -sec trials, respectively.

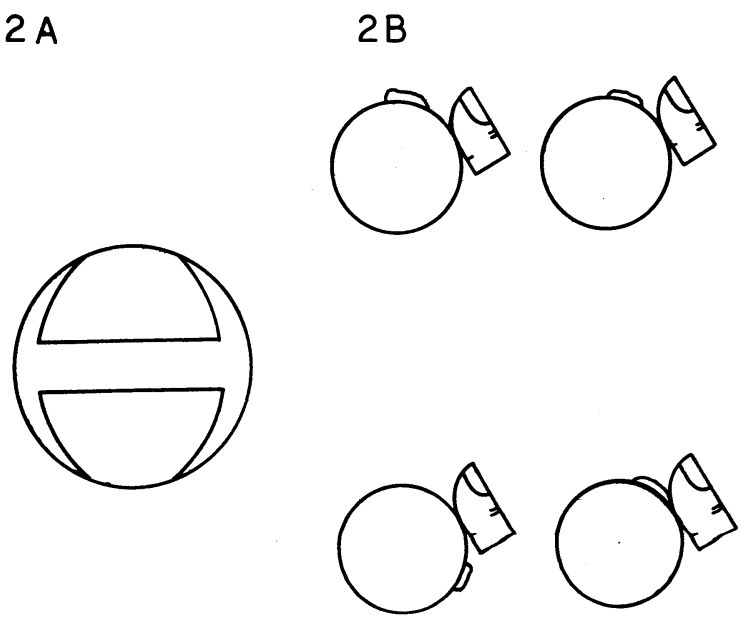

Figure 2. (A) A schematic drawing of the laminate used as a stimulus in the bump illusion and (B) drawings of this illusion produced by four subjects. 
The ridge illusion mean should be halved to make it comparable to the bump illusion, as the magnitude measured in the ridge illusion included two illusory surfaces. When this is done, the ridge magnitude becomes $4.98 \mathrm{~mm}$, which is comparable in size to the magnitude of the bump illusion. The differences in magnitude between the Jones et al. measurements and the present measurements probably are attributable to the measurement techniques employed. Jones et al. used a haptic judgment task in which the subjects reproduced the apparent thickness of the stimulus by making a motor response with the finger of their free hand, whereas in the present study, Von Békésy's (1967) drawing technique was used. Drawing data were obtained in Jones et al., which suggested that the drawing measurement technique yields considerably larger measurements than the haptic technique.

The magnitude of the bump illusion increased over time, but this increase was not statistically reliable. The failure to find a significant effect may be due to two factors. First, the drawing measurement technique produces highly variable responses. Second, the subject's thumb was in contact with the laminate for a few seconds before rotation was started. Kenshalo (1971) reports that adaptation is complete in $5 \mathrm{sec}$ for a light $(10-\mathrm{g})$ weight and takes $25 \mathrm{sec}$ for a heavier weight $(90 \mathrm{~g})$. Therefore, adaptation may have been nearly complete after $5 \mathrm{sec}$ of rotation of the laminate in the bump illusion, and therefore, the 5 -sec measurement represents a near-asymptotic magnitude of the illusion.

Our preferred explanation for these illusions is differential adaptation because the Jones et al. (1974) study showed clear increases in the illusion over time, increases that strongly resemble the time course of pressure adaptation (Kenshalo, 1971). It is probable that other mechanisms, such as lateral inhibition and funneling, influence the magnitude of the illusion (Von Békésy, 1967), but we believe that the illusions are probably caused by differential adaptation to pressure.

\section{REFERENCES}

Cormack, R. H. Haptic illusion: Apparent elongation of a disk rotated between the fingers. Science, 1973, 179, 590-592.

Jones, K. N., Touchstone, R. M., \& Gettys, C. F. A tactile illusion: The rotating hourglass. Perception \& Psychophysics, 1974, 15, 335-338.

Kenshalo, D. R. The cutaneous senses. In J. W. Kling \& L. A. Riggs (Eds.), Woodworth and Schlosberg's experimental psychology. New York: Holt, Rinehart, \& Winston, 1971.

VoN BÉ KÉSY, G. Sensory inhibition. Princeton, N.J: Princeton University Press, 1967.

(Received for publication January 7, 1980.) 